\title{
Ultrasound Needle Visibility in Contrast Mode Imaging: An In Vitro and Ex Vivo Study
}

\section{(ㅇ)(ㅇ) \\ Authors \\ Marga B. Rominger ${ }^{1}$, Katharina Martini ${ }^{1}$, Evelyn Dappa ${ }^{1}$, 2, 3, Gilbert \\ Puippe $^{1}$, Volker Klingmüller ${ }^{1}$, Thomas Frauenfelder ${ }^{1}$, \\ Sergio J. Sanabria ${ }^{3}$ \\ Affiliations \\ 1 University Hospital Zurich, University of Zurich, Institute of Diagnostic and Interventional Radiology, Zurich, Switzerland \\ 2 University Hospital Mainz, Department of Diagnostic and Interventional Radiology, Mainz, Germany \\ 3 ETH, Computer Vision Laboratory, Zurich, Switzerland}

Key words

ultrasound, biopsy, contrast agents

received 24.05.2016

revised 21.12.2016

accepted 07.01.2017

Bibliography

DOI http://dx.doi.org/10.1055/s-0043-101511

Ultrasound Int Open 2017; 3: E82-E88

(c) Georg Thieme Verlag KG Stuttgart · New York

ISSN 2199-7152

Correspondence

Prof. Dr. med. Marga Rominger

UniversitätsSpital Zürich

Institute of Diagnostic and Interventional Radiology

Rämistrasse 100

8091, Zürich

\author{
Switzerland \\ Tel.: + 41/442/551 111 \\ Marga.Rominger@usz.ch
}

\section{ABSTRACT}

Purpose To evaluate needle visibility in ultrasound under contrast mode conditions.

Materials and Methods Needle visibility was evaluated for bevel, EchoTip ${ }^{\circledR}$ and shaft of $18 \mathrm{G}$ Chiba biopsy needle with a $9 \mathrm{MHz}$ linear probe (GE Logiq E9). Insertion angles varied between $30^{\circ}$ (steep) and $90^{\circ}$ (parallel to the probe surface). The acoustic output varied from $5-28 \% .2$ different contrast mode presets with either 'Amplitude Modulation' (Penetration) or 'Phase Inversion Harmonics' (High Resolution) were assessed. All other imaging parameters were kept constant. The visibility of bevel, EchoTip ${ }^{\circledR}$ and shaft was assessed for grayscale and color-coded images with a 3-point Likert-like scale (not, poorly, well visible) by 2 independent readers. The echogenicity of the needle bevel, EchoTip ${ }^{\circledR}$ and shaft was assessed in deciBel ( $\mathrm{dB}$ ) on the color-coded images.

Results With the parallel insertion angle, all needle areas were well visible. With steep insertion the EchoTip ${ }^{\circledR}$ was the only visible area. High Resolution was superior to Penetration $(p<0.001)$. The visibility and echogenicity of the needle bevel $\left(r_{\text {grayscale }}=0.109, \mathrm{p}_{\text {grayscale }}=0.178\right.$; $r_{\text {color-coded }}=0.236$, p color-coded $\left.=0.266 ; r_{\mathrm{dB}}=0.956, \mathrm{p}_{\mathrm{dB}}=0.001\right)$, EchoTip ${ }^{\circledR}$ $\left(r_{\text {grayscale }}=0.477, \mathrm{p}_{\text {grayscale }}=0.018 ; r_{\text {color-coded }}=0.540, \mathrm{p}_{\text {color-coded }}=0.006\right.$; $\left.r_{\mathrm{dB}}=0.911, \mathrm{p}_{\mathrm{dB}}=0.001\right)$ and shaft $\left(r_{\text {grayscale }}=0.124, \mathrm{p}_{\text {grayscale }}=0.563\right.$; $\left.r_{\text {color-coded }}=0.061, p_{\text {color-coded }}=0.775 ; r_{\mathrm{dB}}=0.926, \mathrm{p}_{\mathrm{dB}}=0.001\right)$ increased with increasing acoustic output. Grayscale images were superior to color-coded images for needle visibility $(p=0.004)$.

Conclusion Parallel needle insertion, use of an echogenic tip, adequate choice of presets, increased acoustic output, and dual view of grayscale and color-coded images improve needle visibility in ultrasound under contrast mode conditions.

\section{Introduction}

Ultrasound-guided interventions have proven to be safe and cost-effective procedures, with an increasing number of ultrasound-guided needle biopsies being performed worldwide [1]. To guarantee a successful intervention, needle visibility is paramount. Various factors may influence needle visibility. Numerous studies describe the influence of needle insertion angle and different needle types on needle visibility in B-mode [2-5]. However, these studies mostly examined qualitative assessment based on a visual rating system.

Contrast-enhanced ultrasound is a relatively new imaging technique with a growing number of clinical applications [6-8]. Ultrasound contrast agents contain microbubbles and can be used to detect and characterize lesions of various visceral organs with some lesions best or only visible in the contrast mode [7]. If lesions are better or only visualized after contrast enhancement, ultrasound-guided interventions should be performed under contrast-enhanced conditions. Interestingly, only a few studies so far have evaluated needle visibility in the contrast mode. Sartori et al. and Cao et al. showed that contrast-enhanced ultrasound can have a positive effect as guidance for transthoracic biopsy $[9,10]$. However, needle visibility under contrast-enhanced conditions has not been analyzed.

Needles are more easily detected in fluid and tissues with low echogenicity than in highly echogenic tissues. Contrast-enhanced ultrasound increases the echogenicity of vascularized background tissue and possibly target lesions. Thus, needle visibility may be restricted. A prerequisite of contrast-enhanced ultrasound is the use of harmonic imaging to measure the non-linear acoustic effects of ultrasound interaction with microbubbles as well as a low acoustic output (transmit signal level) to allow continuous imaging without 
destroying the bubbles. Harmonic imaging uses higher harmonic frequencies generated by the tissue as the sound travels through the body. While providing better diagnostic image quality [11], harmonic imaging techniques as well as low acoustic output may decrease needle visibility [12].

The purpose of this study was to qualitatively and quantitatively evaluate the visibility of the needle bevel, echogenic tip and shaft in ultrasound at different insertion angles and contrast mode conditions.

\section{Materials and Methods}

\section{Experimental setting}

We used a tank (diameter $15 \mathrm{~cm} \times 10 \mathrm{~cm} \times 9 \mathrm{~cm}$ ) coated with thin sponges to avoid reflection from the walls. We used $0.9 \%$ saline solution (B. Braun Medical AG, Seesatz 17, 6208 Sempach, Switzerland) tinted with microbubbles (SonoVue ${ }^{\circledR}$, Bracco Suisse S.A. 31, Route de la Galaise, 1228 Plan-les-Quates, Genève Switzerland) to a level with an acoustic deciBel (dB) similar to the human liver based on our clinical database to mimic vascularized background tissue ( $-50 \mathrm{~dB}$ to $-40 \mathrm{~dB}$ ). For ultrasound imaging a linear $9 \mathrm{MHz}$ probe (Logiq E9, GE Medical Systems, Glattbrugg, Switzerland) was mounted on a fixed rack. A self-developed needle positioning system was placed in the tank. The positioning system allowed continuous variable angles holding the needle bevel at the center of rotation with a constant distance to the linear probe ( $\nabla$ Fig. 1$)$. We used an 18G Chiba biopsy needle (Cook Medical Inc., Bloomington, Indiana, USA) with its internal stylet in position. The needle has 3 different areas: bevel at the tip, EchoTip ${ }^{\circledR}$ zone with a length of $7 \mathrm{~mm}$ behind it and shaft ( $\triangleright$ Fig. 2). The EchoTip ${ }^{\circledR}$ zone contains hundreds of microscopic dimples on the needle's surface to ensure strong ultrasound reflection. The shaft was cut to allow $90^{\circ}$ rotation and a parallel position within the tank with respect to the ultrasound probe. The bevel of the needle was always adjusted to the surface of the ultrasound probe to ensure optimal ultrasound reflection. The angles were varied from $30^{\circ}$ (steepest possible angle in combination with the linear probe) to $90^{\circ}$ (parallel to the surface of the linear probe). Finally, porcine muscle from the slaughterhouse was examined to exemplarily demonstrate the effect of contrast mode presets, increased acoustic output and insertion angle on needle visibility.

\section{Ultrasound imaging}

All imaging was performed in the contrast mode. For each variable setting a screenshot of the grayscale (B-mode image with reduced acoustic output) and color-coded (contrast-enhanced) images, as well as a short cine loop of the color-coded images were obtained. The focus was constantly kept at the level of the needle bevel. 2 different contrast mode presets at different angles $(30,45,60,75$, and $90^{\circ}$ ) were tested:

Penetration: based on amplitude modulation,

High Resolution: based on phase inversion harmonics.

Other system settings were adjusted to typical values and kept constant. Thereafter, the acoustic output was varied between 5 and $28 \%$ at different angles $\left(30,60\right.$ and $\left.90^{\circ}\right)$ using the High Resolution preset.

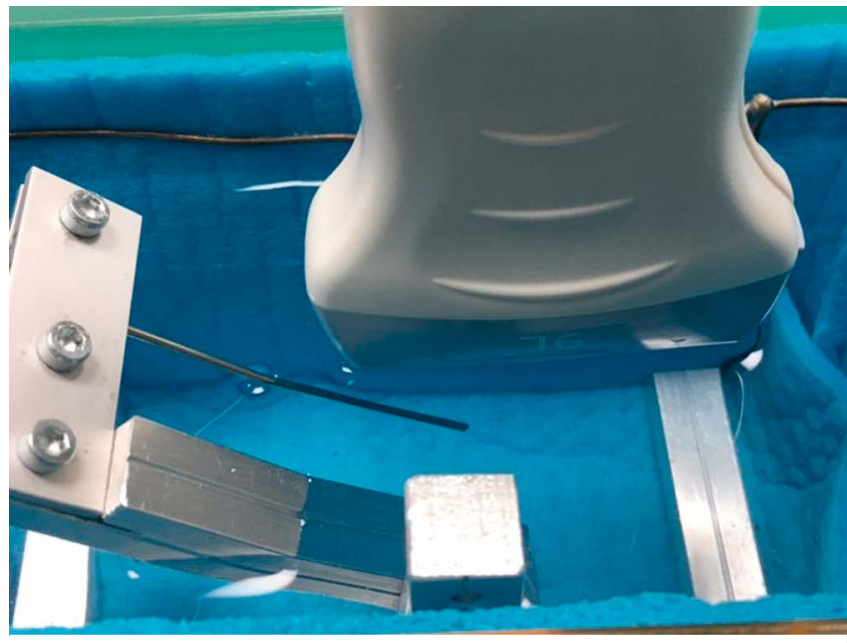

- Fig. 1 Experimental setup of the water tank phantom showing a shorted $18 \mathrm{G}$ Chiba biopsy needle mounted to the positioning system. The linear probe above is fixed to a rack. To mimic the contrast-enhanced background, a $0.9 \%$ saline solution tinted with microbubbles was used.

a

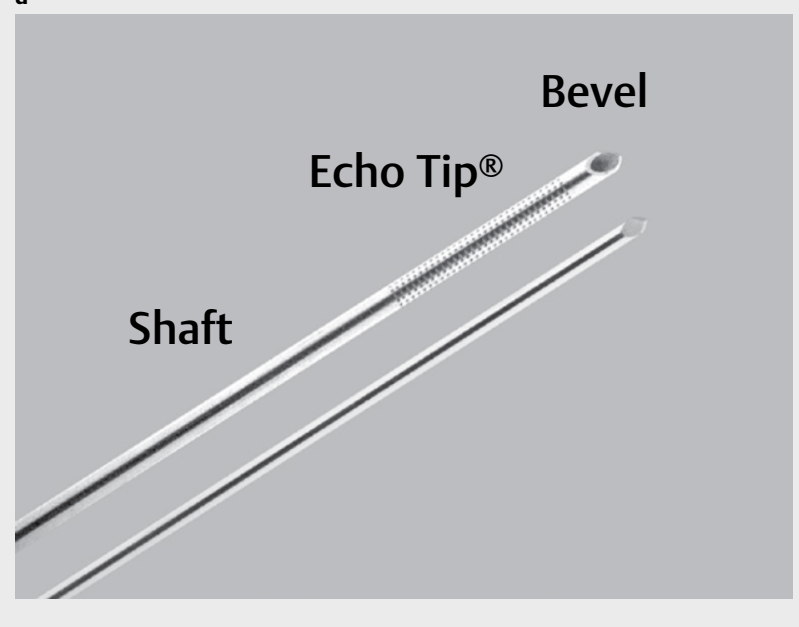

b

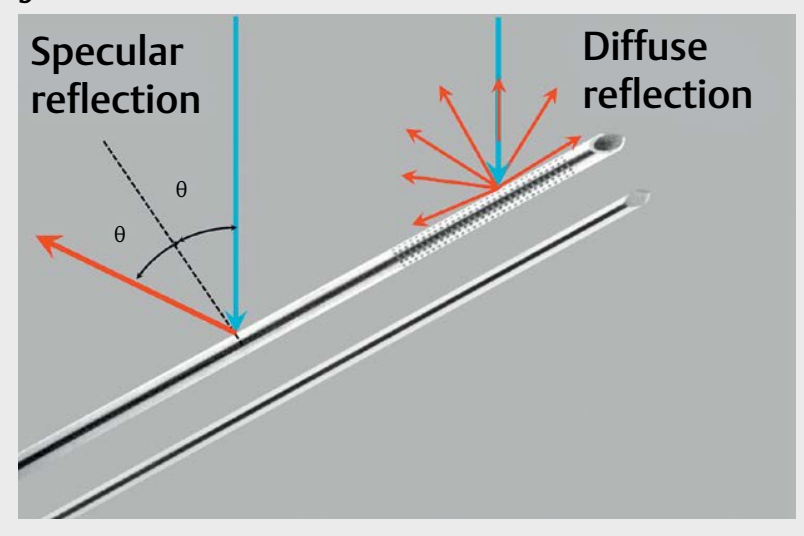

- Fig. 2 a Detail view of the $18 \mathrm{G}$ Chiba biopsy needle shows a) bevel, followed by EchoTip ${ }^{\circledR}$ and shaft and $\mathbf{b}$ different reflection characteristics of EchoTip ${ }^{\circledR}$ and shaft. The lower needle is the internal stylet. Picture: Permission for use granted by Cook Medical, Bloomington, Indiana 


\section{Qualitative and quantitative assessment of the visibility of needle areas}

Qualitative assessment: The images were randomized with the Unix-tool/dev/urandom. The technical data was cropped from the images. 2 radiologists independently assessed the randomized pictures for needle bevel, EchoTip ${ }^{\circledR}$ and shaft using a 3-point Likert-like scale:

(1) needle bevel/EchoTip ${ }^{\circledR} /$ shaft not visible

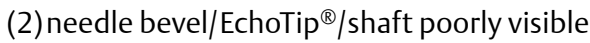

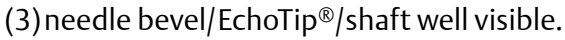

The average of both independent readers was used for further evaluation. In the case of minor differences (not visible vs. poorly visible or poorly visible vs. well visible), the average was used. In the case of major differences (not visible vs. well visible), the study protocol foresaw revisiting and consensus solution. Yet, major differences did not occur.

Quantitative assessment: Manufacturer's time intensity curves for color-coded cine loops were used for quantitative assessment of echo density in $\mathrm{dB}$. Separate region-of-interest measurements for needle bevel, EchoTip ${ }^{\circledR}$, shaft, and background were repeated 3 times and performed for each variable setting. All measurements rendered negative $\mathrm{dB}$ values. Echogenicity increased with less negative values (logarithmic scale represents recorded ultrasound signal level).

\section{Statistical analysis}

Statistical analyses were conducted using Excel (Excel 2016, Microsoft, Redmond, WA). Continuous variables were expressed as mean \pm standard deviation (SD) while categorical variables were expressed as frequencies or percentages. Visibility scores were noted. Mean and standard deviations of $\mathrm{dB}$ measurements were evaluated.

To assess correlations between the different settings, Pearson correlation was used. A correlation coefficient $r>0$ indicates a positive correlation between 2 variables, whereas a correlation coefficient $r<0$ indicates a negative correlation between the 2 tested variables. A 2-sided paired t-test was used to test for significant differences between qualitative image analyses for needle visibility. A $p$-value of $p<0.05$ was considered significant.

\section{Results}

\section{Comparison of contrast mode presets penetration and high resolution at different insertion angles}

In the qualitative assessment the High Resolution preset scored better than the Penetration preset at steep angles $\left(30-60^{\circ}\right)$ ( $>$ Fig. 3a, b) $\left(p_{\text {grayscale }}=0.056\right.$, $\left.p_{\text {color-coded }}=0.012\right)$. Looking at the color-coded images, the entire needle was invisible at steep angles in the penetration mode ( $\mathbf{F i g .} \mathbf{3 b}$ and $\triangleright$ Fig. 4 ). With the High Resolution preset, the EchoTip ${ }^{\circledR}$ became visible ( $\triangleright$ Fig. 3b). Quantitative analysis showed that fewer negative $\mathrm{dB}$ values were obtained with the High Resolution preset for the EchoTip ${ }^{\circledR}$ at steep angles $\left(30-60^{\circ}\right.$ ) compared to the Penetration preset indicating higher echogenicity at High Resolution for the EchoTip ${ }^{\circledR}\left(p<0.001\right.$ for $30-60^{\circ}$ insertion angles High Resolution vs. Penetration). Thus, the echogenicity of the EchoTip ${ }^{\circledR}$ was superior to the echogenicity of the contrast-enhanced background ( $-\mathrm{dB} 50$ to $-\mathrm{dB} 40$ ). With an increasing insertion angle, the echogenicity of the needle bevel $(p<0.001)$, EchoTip $^{\circledR}(p<0.001)$, and shaft $(p<0.001)$ increased for both presets ( $\triangleright$ Fig. 5).

\section{Influence of acoustic output at different insertion angles}

An increasing acoustic output improved visibility scores $\left(r_{\text {gray- }}\right.$ scale $=0.109, p_{\text {grayscale }}=0.178 ; r_{\text {color-coded }}=0.236$, $\left.p_{\text {color-coded }}=0.266\right)$, EchoTip $^{\circledR}\left(r_{\text {grayscale }}=0.477, \mathrm{P}_{\text {grayscale }}=0.018 ; r_{\text {color-coded }}=0.540\right.$, $\left.\mathrm{P}_{\text {color-coded }}=0.006\right)$ and shaft $\left(r_{\text {grayscale }}=0.124, \mathrm{P}_{\text {grayscale }}=0.563\right.$; $\left.r_{\text {color-coded }}=0.061, P_{\text {color-coded }}=0.775\right)$. At steep angles of $30-60^{\circ}$, the EchoTip ${ }^{\circledR}$ scored best ( $\triangleright$ Fig. 6). The dB values of all needle areas at all angles improved with an increasing acoustic output ( $>$ Fig. 7) ( $\mathrm{p}_{\text {bevel }}=0.001, \mathrm{p}_{\text {Echotip }}{ }^{\circledR}=0.001, \mathrm{p}_{\text {shaft }}=0.001$ ).

\section{Comparison of grayscale and color-coded images}

Overall, needle visibility was better on the grayscale images than the color-coded images (mean score 2098 vs. 2311 and an SD of 0.962 and 0.798 , respectively; $p=0.004)$ ( $\triangleright$ Fig. 3,6 ).

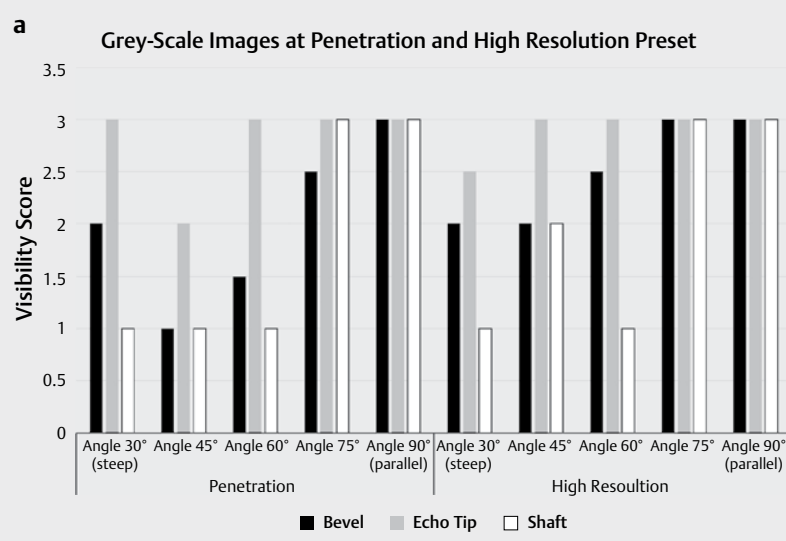

b 3.5 Color-Coded Images at Penetration and High Resolution Preset

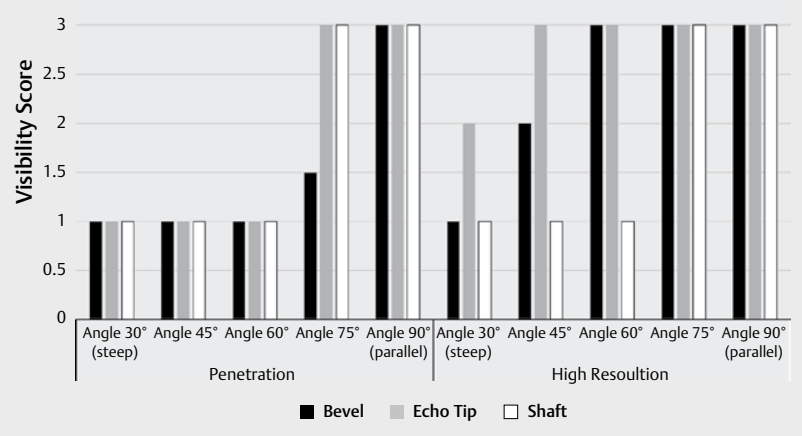

- Fig. 3 a Visibility scoring of Penetration and High Resolution preset. On a grayscale images visibility scores of the High Resolution preset was on average equal or superior to the Penetration preset. On b color-coded images the entire needle was invisible with an insertion angle from $30^{\circ}$ to $60^{\circ}$ using Penetration, whereas with High Resolution the EchoTip ${ }^{\circledR}$ was visible. Visibility scores: $1=$ needle not visible, 2 = poorly visible, and 3 = well visible. A constant acoustic output of $10 \%$ was used. 


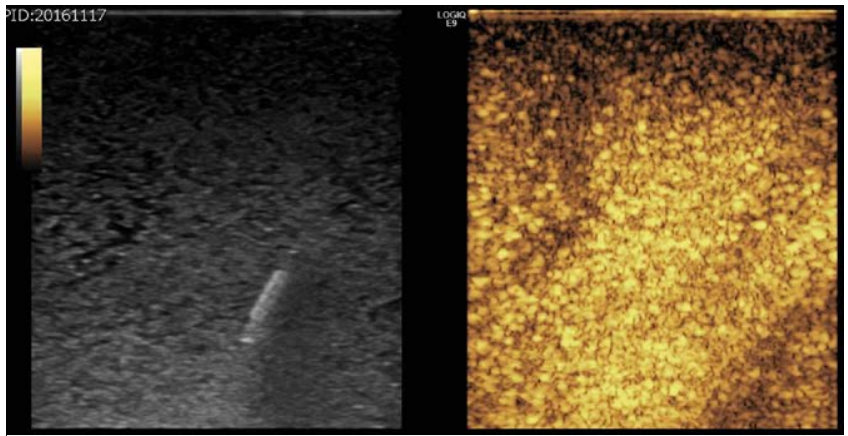

- Fig. 4 Dual contrast mode view of the $18 \mathrm{G}$ Chiba biopsy needle at an insertion angle of $30^{\circ}$ with Penetration preset, $10 \%$ acoustic output and microbubble solution as background tissue. On the grayscale image on the left, the needle bevel and EchoTip ${ }^{\circledR}$ are visible. On the color-coded image on the right side, the entire needle is not visible due to the contrast-enhanced background tissue.

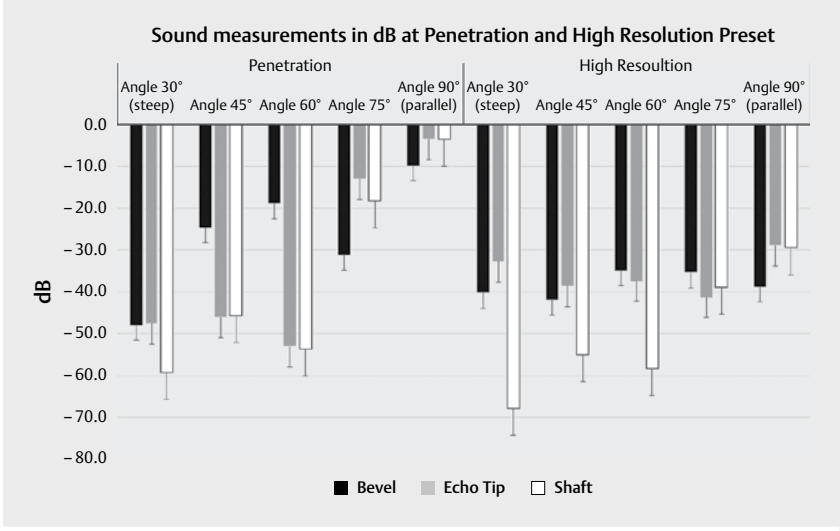

- Fig. 5 Ultrasound measurements in needle region-of-interest show higher ultrasound reflection (recorded signal level in $\mathrm{dB}$ ) for EchoTip ${ }^{\circledR}$ between $30^{\circ}$ and $60^{\circ}$ for the High Resolution preset explaining the superior visibility scores of $\mathbf{\nabla} \mathbf{F i g}$. $\mathbf{3} \mathbf{b}$ for this preset. As a reference, the level of the contrast-enhanced background tissue was between $-\mathrm{dB} 50$ and $-\mathrm{dB} 40$. The acoustic output was $10 \%$.

\section{Porcine muscle from the slaughterhouse}

The effect of contrast mode preset, acoustic output and insertion angle is exemplarily demonstrated with porcine muscle from the slaughterhouse in $\mathbf{F i g . ~} \mathbf{8}$. On both the grayscale and color-coded image, the use of the High Resolution preset, increased acoustic output and parallel insertion angle improved needle visibility.

\section{Discussion}

Our in-vitro phantom data indicates that parallel insertion with respect to the probe gives best results for needle visibility in contrast mode imaging. If this cannot be achieved, for example in the case of a deep liver lesion, the use of an echogenic tip and increased acoustic output can improve needle visibility. The High Resolution preset scored better than the Penetration preset in regard to needle visibility for steep angles in this study. Grayscale images scored better than the color-coded images for needle visibility.

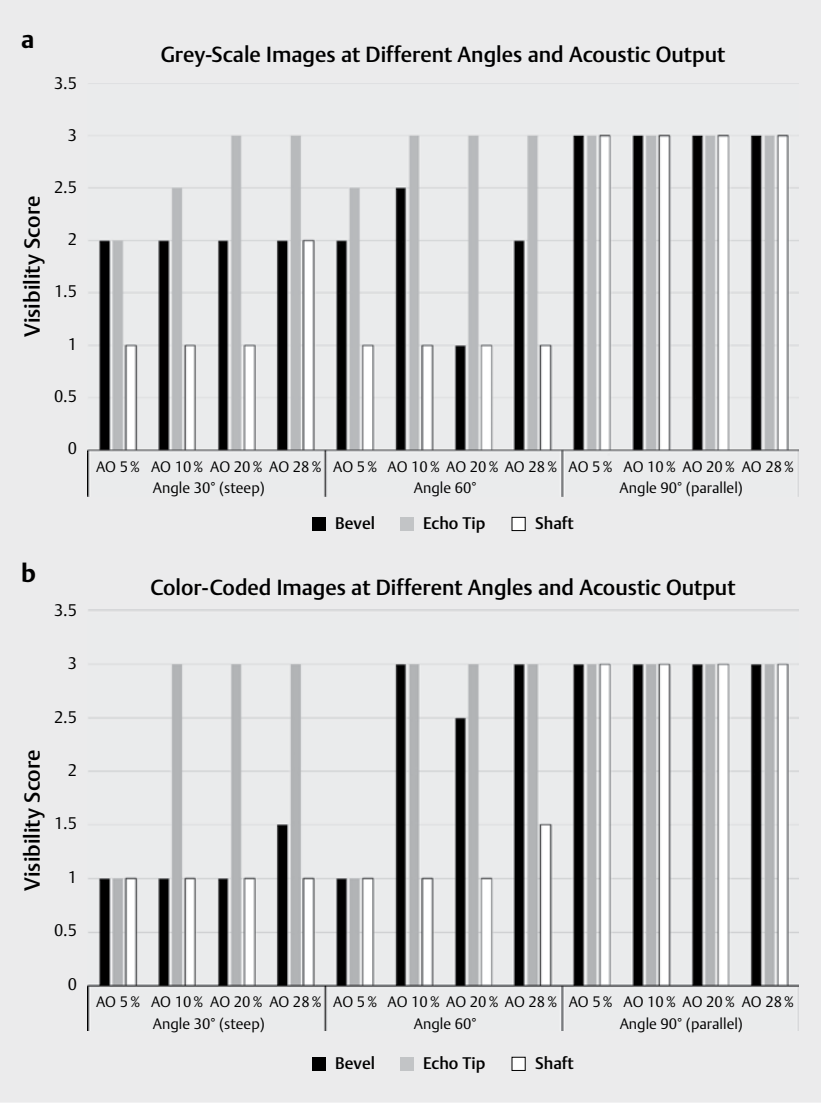

- Fig. 6 a Comparison of different needle insertion angles and acoustic output at High Resolution preset: on grayscale $\mathbf{a}$ and color-coded $\mathbf{b}$ images increased acoustic output and angles increased needle visibility. Visibility scores: 1 = needle not visible, 2 = poorly visible, and $3=$ well visible.

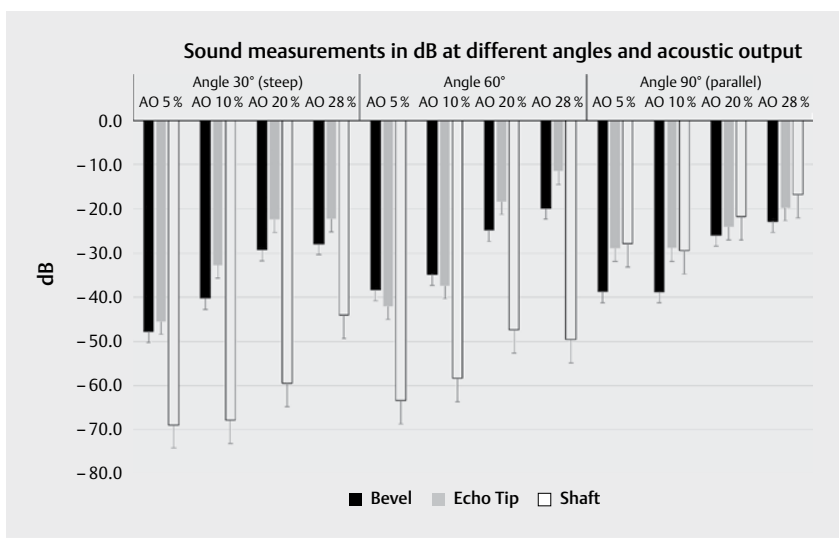

- Fig. 7 Ultrasound measurements in needle region-of-interest show higher ultrasound reflection (recorded signal level in $\mathrm{dB}$ ) of needle bevel, EchoTip ${ }^{\circledR}$ and shaft with increasing acoustic output $\mathrm{AO}$ ) and insertion angles. The highest $\mathrm{dB}$ values were obtained for the needle shaft parallel to the linear probe. The High Resolution preset was used.

The correlation between different needle types, insertion angles and needle visibility has been a subject of extensive research in conventional B-mode. In contrast to these studies, we examined 

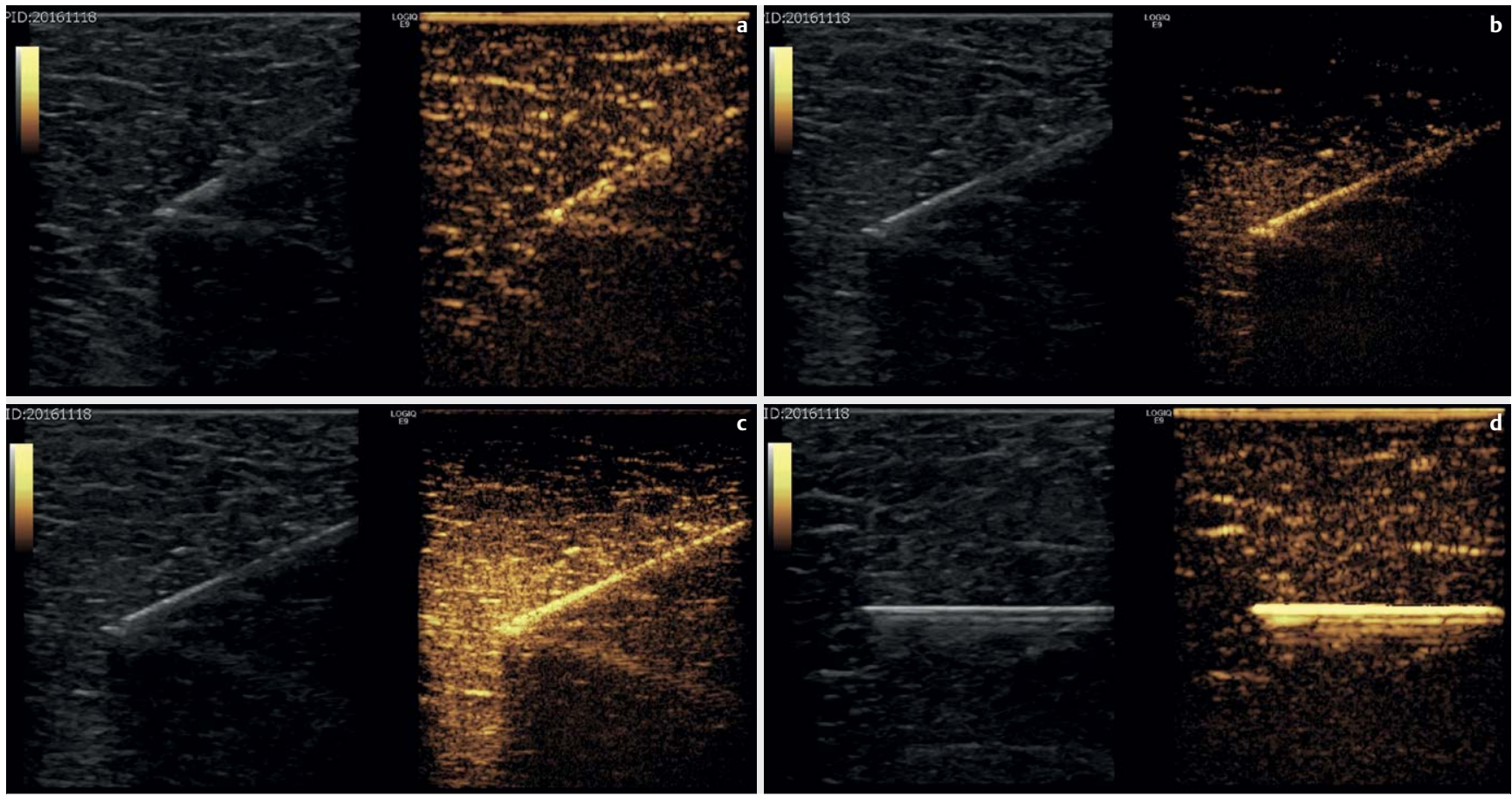

- Fig. 8 a Dual contrast mode view of porcine muscle exemplarily shows a Penetration preset at an insertion angle of $60^{\circ}$ with an acoustic output of $10 \%$, b improved needle visibility with the High Resolution preset, $\mathbf{c}$ further improvement of needle visibility with increased acoustic output of $20 \%$, and $\mathbf{d}$ best needle visibility with parallel insertion angle. The Penetration preset with an acoustic output of $10 \%$ was used. Other system settings were kept constant.

the needle visibility of grayscale images (B-mode images with reduced acoustic output) and color-coded (contrast-enhanced) images presented as dual view in contrast mode. In a study of Cao et al., contrast-enhanced ultrasound was used to evaluate pulmonary and mediastinal lesions and to select a biopsy route. The biopsy itself was performed in conventional B-mode [10]. In a case review of Sartori et al., a biopsy of a peripheral lung lesion was performed under contrast mode conditions. No special mention was made regarding the settings used in contrast mode [9]. In agreement with previous studies performed with conventional B-mode [13-15], we found that the needle insertion angle greatly affects needle visibility in contrast mode as well. The major improvement of needle visibility was achieved in this study with an insertion angle greater than $60^{\circ}$. At an angle greater than $60^{\circ}$ there was sufficient return of echoes for all needle zones, especially the shaft. The smooth metallic surface of the shaft is a specular (mirror-like) reflector of ultrasound waves. For specular reflection, the angle at which the wave hits the surface equals the angle at which it is reflected ( $\mathbf{F i g . ~} \mathbf{2 b}$ ). Thus, within an echogenic background the needle or part of the needle may become invisible at steep insertion angles ( $>$ Fig. 4).

Many authors agree that needles with a special echogenic design improve needle visibility on conventional B-mode ultrasound $[3,4,16]$. The results of our study illustrate the importance of an echogenic needle tip design to improve visibility within an echogenic background at steep insertion angles on contrast mode. At steep needle insertion angles, the EchoTip ${ }^{\circledR}$ was visible, whereas the needle shaft was not or only barely visible. Starting from conventional metallic designs, needle manufacturers have improved the visibility of the needle tip in ultrasound sonography by embedding microstructures that behave as a dense cloud of scatterers. Similar to a hyperechogenic tissue, these microstructures ideally lead to a diffuse ultrasound reflection, which is isotropic (direction-independent) regardless of the needle insertion angle ( $\vee$ Fig. 2b). For instance, the EchoTip ${ }^{\circledR}$ zone contains hundreds of randomly located microscopic dimples [17] ( Fig. 2a).

The readers found better needle visibility with increasing acoustic output for grayscale and color-coded images. The quantitative analysis of color-coded images confirmed increased echogenicity for increasing acoustic output. Yet, for implementation in a clinical setting, it should be considered that microbubbles can be destroyed at a higher acoustic output. Most clinical investigators report that a high acoustic output ( $>50 \%$ ) leads to the destruction of microbubbles [18], although some authors suggest that the acoustic output as displayed on clinical ultrasound scanners is not a reliable indicator [19]. A low acoustic output technique allows for continuous scanning without early destruction of the microbubbles [6]. Therefore, we conclude that a moderate increase of the acoustic output likewise from $10 \%$ to $20 \%$ is suitable for positively influencing needle visibility.

Contrast-enhanced ultrasound examinations were normally performed with a dual grayscale and color-coded contrast presentation. We found that the needle visibility of the grayscale image was superior to the color-coded image. Thus, dual view should be helpful for monitoring needle placement under contrast mode conditions.

Similar to a B-mode image, the grayscale image shows the linear component, which facilitates needle guidance [15]. 
The contrast mode presets for the color-coded images in this study work with 2 different imaging techniques: 'amplitude modulation' (Penetration) and 'phase inversion harmonics' (High Resolution). Both techniques use the non-linear acoustic behavior of microbubbles for enhancing microbubbles and suppress signals from the surrounding tissue. With 'phase inversion harmonics' [20], 2 ultrasound pulses, dephased by $180^{\circ}$ (inverse sign), are consecutively emitted. The dimensional oscillations of gas microspheres contained in the contrast agents generate non-linear echoes (e. g., a quadratic response). The machine software highlights these echoes by adding up the 2 harmonic waves. Thus, while linear echoes generated by surrounding tissues are mutually cancelled, non-linear echoes from contrast agents generate a strong signal. 'Amplitude modulation', on the other hand, includes the transmission of pulses with a different amplitude to enhance the non-linear signal from microbubbles. When the echoes are received, the amplitude difference will be compensated before the second echo signal is subtracted from the first one, to remove the linear scattering. The advantage of 'amplitude modulation' is the ability to detect pressure-dependent non-linear effects. However, 'amplitude modulation' has been reported to show practical difficulties with respect to 'phase inversion harmonics' to achieve sufficient cancellation of linear tissue signal [21]. We found that the echogenicity and visibility of the EchoTip ${ }^{\circledR}$ was better with High Resolution than Penetration at a steep insertion angle. Thus, with High Resolution the echogenicity of the EchoTip ${ }^{\circledR}$ was higher than the background and became visible. Yet, within a low echogenic background, for example in the case of a non-enhancing lesion or abscess, the echogenicity of the Penetration preset might be sufficient to visualize the needle.

Experiments were conducted in a tank, with microbubble-enhanced isotonic saline solution serving as the "background tissue". The advantage of measuring in such a phantom is the elimination of interference from other impedance differences and the allowance for standardized, reproducible measurements. The disadvantage is, as with most in-vitro models, that the results cannot be simply transferred to the human body and the clinical routine. Background noise from surrounding body tissue could have an additional and variable influence on needle visibility. However, the comparison of in vitro results with exemplary results in a highly heterogeneous tissue (ex vivo muscle) showed consistent needle visibility trends in this study. We limited our experiments to one vendor and used a linear probe to exclude an influence of curved array on angulation. Although the principle of contrast-enhanced ultrasound is well established, different ultrasound machine vendors use different hardware and image reconstruction algorithms, as well as different settings, which may influence results. As an example, in this study the performance of 2 non-linear imaging algorithms "amplitude modulation" and "phase inversion harmonics" differed. In general, ultrasound machines are closed systems and insights to the post-processing of raw data are limited.

In conclusion, contrast mode presets may influence needle visibility. Higher acoustic output improves needle visibility in ultrasound under contrast mode conditions. The EchoTip ${ }^{\circledR}$ was especially helpful for steep needle insertion angles, due to the isotropic ultrasound reflection. Shaft visibility improved significantly with parallel or near parallel insertion angles, whereas for steep insertion angles reflection away from the insonification direction reduc- es the measured ultrasound echo. The dual view is recommended because the needle visibility of the grayscale image, which shows a linear response similar to conventional B-mode, was superior to that of the color-coded image, which represents non-linear harmonic phenomena excited by microbubbles.

\section{Conflict of Interest}

The authors declare that they have no conflict of interest.

\section{References}

[1] Barr RG. Improved needle visualization with electronic beam steering: proof of concept. Ultrasound Q 2012; 28: 59-64

[2] Kawai N, Minamiguchi H, Sato M, Nakai M, Sanda H, Tanaka T et al. Evaluation of vascular puncture needles with specific modifications for enhanced ultrasound visibility: In vitro study. J Radiol 2012; 4: 273-277

[3] Sviggum HP, Ahn K, Dilger JA, Smith HM. Needle echogenicity in sonographically guided regional anesthesia: blinded comparison of 4 enhanced needles and validation of visual criteria for evaluation. J Ultrasound Med 2013; 32: 143-148

[4] Abbal B, Choquet O, Gourari A, Bouic N, Massone A, Biboulet P et al. Enhanced visual acuity with echogenic needles in ultrasound-guided axillary brachial plexus block: A randomized, comparative, observer-blinded study. Minerva Anestesiol 2015; 81: 369-378

[5] Miura M, Takeyama K, Suzuki T. Visibility of ultrasound-guided echogenic needle and its potential in clinical delivery of regional anesthesia. Tokai J Exp Clin Med 2014; 39: 80-86

[6] Chung YE, Kim KW. Contrast-enhanced ultrasonography: advance and current status in abdominal imaging. Ultrasonography 2015; 34: 3-18

[7] Malhi H, Grant EG, Duddalwar V. Contrast-enhanced ultrasound of the liver and kidney. Radiol Clin North Am 2014; 52: 1177-1190

[8] Lackey L, 2nd, Peterson C, Barr RG. Contrast-enhanced ultrasound-guided radiofrequency ablation of renal tumors. Ultrasound $Q$ 2012; 28: 269-274

[9] Sartori S, Nielsen I, Trevisani L, Tombesi P, Ceccotti P, Abbasciano V. Contrast-enhanced sonography as guidance for transthoracic biopsy of a peripheral lung lesion with large necrotic areas. J Ultrasound Med 2004; 23: 133-136

[10] Cao BS, Wu JH, Li XL, Deng J, Liao GQ. Sonographically guided transthoracic biopsy of peripheral lung and mediastinal lesions: role of contrast-enhanced sonography. J Ultrasound Med 2011; 30: 1479-1490

[11] Choudhry S, Gorman B, Charboneau JW, Tradup DJ, Beck RJ, Kofler JM et al. Comparison of tissue harmonic imaging with conventional US in abdominal disease. Radiographics 2000; 20: 1127-1135

[12] Mesurolle B, Bining HJ, El Khoury M, Barhdadi A, Kao E. Contribution of tissue harmonic imaging and frequency compound imaging in interventional breast sonography. J Ultrasound Med 2006; 25: 845-855

[13] Hopkins RE, Bradley M. In-vitro visualization of biopsy needles with ultrasound: a comparative study of standard and echogenic needles using an ultrasound phantom. Clin Radiol 2001; 56: 499-502

[14] Schafhalter-Zoppoth I, McCulloch CE, Gray AT. Ultrasound visibility of needles used for regional nerve block: an in vitro study. Reg Anesth Pain Med 2004; 29: 480-488

[15] Chin KJ, Perlas A, Chan VW, Brull R. Needle visualization in ultrasound-guided regional anesthesia: challenges and solutions. Reg Anesth Pain Med 2008; 33: 532-544 
[16] Wiesmann T, Borntrager A, Zoremba M, Neff M, Wulf H, Steinfeldt T. Compound imaging technology and echogenic needle design: effects on needle visibility and tissue imaging. Reg Anesth Pain Med 2013; 38 : 452-455

[17] Nichols K, Wright LB, Spencer T, Culp WC. Changes in ultrasonographic echogenicity and visibility of needles with changes in angles of insonation. J Vasc Interv Radiol 2003; 14: 1553-1557

[18] Hung SH, Yeh CK, Tsai TH, Chen T, Chen RC. A simple method for quantifying ultrasound-triggered microbubble destruction. Ultrasound Med Biol 2011; 37: 949-957
[19] Forsberg F, Shi WT, Merritt CR, Dai Q, Solcova M, Goldberg BB. On the usefulness of the mechanical index displayed on clinical ultrasound scanners for predicting contrast microbubble destruction. J Ultrasound Med 2005; 24: 443-450

[20] Pop CM, Mihu D, Badea R. Role of contrast-enhanced ultrasound (CEUS) in the diagnosis of endometrial pathology. Clujul Med 2015; 88: 433-437

[21] Hofstad E. Ultrasound contrast imaging - improved tissue suppression in amplitude modulation. Norwegian University of Science and Technology. 2006 